\title{
Simultaneous Diophantine approximation and asymptotic formulae on manifolds
}

\author{
by \\ GLyn Harman (Egham)
}

1. Introduction. Write \|\| to denote distance to the nearest integer. Throughout this paper $n$ (without subscript) denotes a positive integer. A central question in the metric theory of Diophantine approximation is to obtain asymptotic formulae for the number of solutions to the inequality

$$
\|\alpha n\|<\psi(n), \quad n \leq N,
$$

for almost all $\alpha$ under suitable conditions on $\psi$, where $\psi(n) \in[0,1 / 2)$ for $n \leq N$. This subject is covered in Chapters 3 and 4 of [9] (see also [10]).

The question has been generalised to simultaneous approximation and approximation to linear forms. In these cases we consider $\mathbf{x}=\left(x_{1}, \ldots, x_{k}\right) \in$ $\mathbb{R}^{k}$ and count solutions to

$$
\max \left(\left\|n x_{1}\right\|, \ldots,\left\|n x_{k}\right\|\right)<\psi(n), \quad n \leq N,
$$

Or

$$
\left\|n_{1} x_{1}+n_{2} x_{2}+\ldots+n_{k} x_{k}\right\|<\psi\left(\max _{j}\left|n_{j}\right|\right), \quad \max _{j}\left|n_{j}\right| \leq N,
$$

or (as later in this paper), we consider (2) with all the $n_{j}$ non-negative. If we write

$$
\Psi_{1}(N)=2^{k} \sum_{n=1}^{N} \psi(n)^{k}, \quad \Psi_{2}(N)=2 k \sum_{n=1}^{N} n^{k-1} \psi(n),
$$

then we expect the number of solutions to (1) and (2) (in the case all $n_{j}$ non-negative) to be asymptotically equal to $\Psi_{1}(N)$ and $\Psi_{2}(N)$ respectively, for almost all $\mathbf{x} \in \mathbb{R}^{k}$.

In general, having more variables makes such problems easier. However, if one restricts $\mathbf{x}$ to a submanifold of $\mathbb{R}^{k}$ then the question becomes much more difficult. As is well known, it is very non-trivial even to prove that the convergence of $\Psi_{1}(\infty)$ implies that there are only finitely many solutions to

2000 Mathematics Subject Classification: 11K60, 11J20. 
(1) for almost all $\mathbf{x}$ on a manifold (see Chapter 9 of [9] for a brief introduction, or [5] for a more thorough discussion). The question of obtaining asymptotic formulae on manifolds has received relatively little attention to date (see $[3,4,7]$ ) and it is the purpose of this paper to address this issue.

Before introducing the notation required to state our main theorems we give a corollary which illustrates the strengths and weaknesses of our results. The rational normal curve $\left(\alpha, \alpha^{2}, \ldots, \alpha^{k}\right)$ has been of fundamental significance in the development of the theory of Diophantine approximation on manifolds and so we use it here as an example.

TheORem 1. Let $\varepsilon>0, k \geq 2$ be given. Then the number of solutions to

$$
\max _{1 \leq j \leq k}\left\|\alpha^{j} n+\beta_{j}\right\|<\left(\frac{\log ^{2+\varepsilon} n}{n}\right)^{1 / k}, \quad n \leq N,
$$

is, for almost all $\alpha \in \mathbb{R}$ and any $\left(\beta_{1}, \ldots, \beta_{k}\right) \in \mathbb{R}^{k}$, asymptotically equal to

$$
2^{k} \sum_{n=1}^{N} \frac{\log ^{2+\varepsilon} n}{n} .
$$

Also, the number of solutions to

$$
\left\|\alpha n_{1}+\alpha^{2} n_{2}+\ldots+\alpha^{k} n_{k}+\beta\right\|<\frac{\log ^{2 k+\varepsilon} n}{n^{k}}, \quad n=\max _{j} n_{j} \leq N,
$$

is asymptotically equal to

$$
2 k \sum_{n=1}^{N} \frac{\log ^{2 k+\varepsilon} n}{n}
$$

for almost all $\alpha$ and any $\beta$.

The reader will note that we are a factor $\log ^{3} n$ away from obtaining a best possible result for (3) and a factor $\log ^{1+2 k} n$ adrift for (4). It is an inherent weakness in our method (which is similar to, but more efficient than the method of [3]) that we cannot obtain the best possible result. The reader should contrast the method of [7] which gives a result $\log n$ away from best possible (one requires $\Psi(N) \gg(\log N)^{1+\delta}$ there in order to obtain a non-trivial result as is made explicit in their Theorem 2), but does not work for the rational normal curve (manifolds there need to be at least two-dimensional in $\mathbb{R}^{k}$ with $k \geq 7$ ). It is conceivable that their method, if suitably refined, would yield a best possible result on certain manifolds. The reader should also note that we have made the problem inhomogeneous. This has not affected the quality of the results since our methods are based on uniform distribution ideas. To date there have been very few inhomogeneous results for Diophantine approximation on manifolds (see [2]). 
We use the following notation from [5] to state our results. Let $\mathfrak{M}$ be a submanifold of $\mathbb{R}^{k}$ with induced Lebesgue measure $\mu$ (see p. 15 of [5]). In the following, whenever we use the phrase "almost all $\mathbf{x} \in \mathfrak{M}$ ", we shall mean "with respect to $\mu$ ". We say that $\mathfrak{M}$ is a Khinchin type manifold for convergence if the convergence of $\Psi_{1}(\infty)$ implies that there are only finitely many solutions to (1) for almost all $\mathbf{x} \in \mathfrak{M}$. Similarly we call $\mathfrak{M}$ a Groshev type manifold for convergence if the convergence of $\Psi_{2}(\infty)$ implies that there are only finitely many solutions to (2) for almost all $\mathbf{x} \in \mathfrak{M}$. We write $S(N, \mathbf{x}, \beta)$ for the number of solutions to

$$
\|\mathbf{x . n}+\beta\|<\psi\left(\mathbf{n}^{*}\right), \quad \mathbf{n}^{*}=\max \left(n_{1}, \ldots, n_{k}\right) \leq N, \mathbf{n} \in \mathbb{N}^{k},
$$

and $T(N, \mathbf{x}, \mathbf{y})$ for the number of solutions to

$$
\max _{1 \leq j \leq k}\left\|n x_{j}+y_{j}\right\|<\psi(n), \quad n \leq N .
$$

Our main results are then as follows.

Theorem 2. Let $\varepsilon>0, k \geq 2$ and $\mathfrak{M} \subset \mathbb{R}^{k}$ a Khinchin type manifold for convergence. Suppose $\psi(n) \in(0,1 / 2)$ is decreasing and satisfies

$$
\psi(n)>\frac{\log ^{1+\varepsilon} n}{n^{k}} .
$$

Then, for almost all $\mathbf{x} \in \mathfrak{M}$, for every $\beta \in \mathbb{R}$, we have, as $N \rightarrow \infty$,

$$
\Psi_{2}(N) \ll S(N, \mathbf{x}, \beta) \ll \Psi_{2}(N) .
$$

Here the implied constants depend on $\varepsilon$ and $\mathbf{x}$ but not on $\beta$. If we suppose that $\psi(n)$ satisfies

$$
\psi(n)>\frac{\log ^{k+1+\varepsilon} n}{n^{k}}
$$

then, as $N \rightarrow \infty$, for almost all $\mathbf{x} \in \mathfrak{M}$,

$$
S(N, \mathbf{x}, \beta) \sim \Psi_{2}(N) .
$$

Suppose, in addition, that for $1 \leq n<m \leq 2 n$ we have

$$
\psi(n)-\psi(m) \ll \frac{m-n}{n} \psi(n) .
$$

Then, for almost all $\mathbf{x} \in \mathfrak{M}$,

$$
S(N, \mathbf{x}, \beta)=\Psi_{2}(N)+O(\Theta(N))
$$

where

$$
\Theta(N)=\sum_{n=1}^{N} \frac{n^{k-1} \psi(n)}{\phi(n)} \text { with } \phi(n)=\left(\frac{n^{k} \psi(n)}{1+(\log n)^{k+1+\varepsilon}}\right)^{1 /(2 k+1)} .
$$

REMARK. An alternative hypothesis to (5) would be " $\phi(n)$ increasing". 
TheOREm 3. Let $\varepsilon>0, k \geq 2$ and $\mathfrak{M} \subset \mathbb{R}^{k}$ a Groshev type manifold for convergence. Suppose $\psi(n) \in(0,1 / 2)$ is decreasing and satisfies

$$
\psi(n)^{k}>\frac{\log ^{1+\varepsilon} n}{n} .
$$

Then, for almost all $\mathbf{x} \in \mathfrak{M}$, for every $\mathbf{y} \in \mathbb{R}^{k}$, we have

$$
\Psi_{1}(N) \ll T(N, \mathbf{x}, \mathbf{y}) \ll \Psi_{1}(N) .
$$

Here the implied constants depend on $\varepsilon$ and $\mathbf{x}$ but not on $\mathbf{y}$. If we suppose that $\psi(n)$ satisfies

$$
\psi(n)^{k}>\frac{\log ^{2+\varepsilon} n}{n},
$$

then, for almost all $\mathbf{x} \in \mathfrak{M}$, for every $\mathbf{y} \in \mathbb{R}^{k}$, we have, as $N \rightarrow \infty$,

$$
T(N, \mathbf{x}, \mathbf{y}) \sim \Psi_{1}(N) .
$$

Suppose, in addition, that for $1 \leq n<m \leq 2 n$ we have

$$
\psi(n)^{k}-\psi(m)^{k} \ll \frac{m-n}{n} \psi(n)^{k} .
$$

Then, for almost all $\mathbf{x} \in \mathfrak{M}$,

$$
T(N, \mathbf{x}, \mathbf{y})=\Psi_{1}(N)+O(\Xi(N))
$$

where

$$
\Xi(N)=\sum_{n=1}^{N} \frac{\psi(n)^{k}}{\lambda(n)} \quad \text { with } \quad \lambda(n)=\left(\frac{n \psi(n)^{k}}{1+(\log n)^{2+\varepsilon}}\right)^{1 /(k+2)} .
$$

Remarks. Theorem 3 improves Theorem 1 of [3]. The condition that $\mathfrak{M}$ be a Khinchin or Groshev type manifold for convergence can be relaxed at the expense of imposing stricter conditions on $\psi$. For example, if the manifold is extremal in the sense that, for every $\varepsilon>0$,

$$
\|\mathbf{x} . \mathbf{n}\|>\mathbf{n}^{*-k-\varepsilon}
$$

has only a finite number of solutions, then (8) holds with $(\log n)^{2+\varepsilon}$ replaced by $n^{\varepsilon}$ in the conditions on $\psi$. To date better results are known for manifolds of Groshev type for convergence than for those of Khinchin type. One could use Theorems 2 and 3 to demonstrate that a manifold of Groshev type for convergence is "almost" of Khinchin type for convergence as well, and vice versa. In fact, it is more efficient to use "Transference Theorems". We leave it to the reader to verify that Theorems 2 and 6 of [6, Chapter 5] lead to the following two results.

TheOREM 4A. Let $\mathfrak{M}$ be a Groshev type manifold for convergence. Then, given $\varepsilon>0$, for almost all $\mathbf{x} \in \mathfrak{M}$ there are only finitely many solutions to

$$
\max \left(\left\|x_{1} n\right\|, \ldots,\left\|x_{k} n\right\|\right)<n^{-1 / k}(\log n)^{-1-\varepsilon} .
$$


THEOREM 4B. Let $\mathfrak{M}$ be a Khinchin type manifold for convergence. Then, given $\varepsilon>0$, for almost all $\mathbf{x} \in \mathfrak{M}$ there are only finitely many solutions to

$$
\|\mathbf{x . n}\|<\mathbf{n}^{*-k}\left(\log \mathbf{n}^{*}\right)^{-k-\varepsilon} .
$$

As an example of the simple principle behind our method we prove first the following theorem which would give a weaker result than Schmidt's work ([12], [9, Chapter 4]) for small $\psi$, but is stronger for the case of larger $\psi$ we state.

TheOREm 5. Let $\varepsilon>0,0<\theta<1$. For almost all real $\alpha$, and any given real $\beta$, the number of solutions to

$$
\|\alpha n+\beta\|<n^{-\theta}, \quad n \leq N,
$$

is, for $N \geq 2$,

$$
\frac{2 N^{1-\theta}}{1-\theta}+O\left(N^{(1-\theta) / 2}(\log N)^{1 / 2+\varepsilon}\right) .
$$

REMARK. We have lowered the exponent of the logarithm here from 2 (in Schmidt's work) to $1 / 2$.

2. Proof of Theorem 5. We will give a lower bound for the number of solutions, the upper bound follows by a similar argument. Write

$$
\psi(n)=n^{-\theta} \text {. }
$$

Let

$$
D_{M}(n \alpha)=\sup _{\gamma \in[0,1)}\left|\sum_{\substack{n \leq M \\\{\alpha n\} \leq \gamma}} 1-\gamma M\right| .
$$

Then it is well known (see [9, Theorem 5.15], the original result goes back to the work of Khinchin [11]) that, for almost all $\alpha$,

$$
D_{M}(n \alpha) \ll(\log M)^{1+\varepsilon} \quad \text { as } M \rightarrow \infty .
$$

We write $\phi(n)$ for an increasing function whose definition will not appear until it can be justified. We split up the range $1 \leq n \leq N$ as follows: $N_{1}=1$, $N_{j}(j>1)$ is the smallest integer exceeding

$$
N_{j-1}\left(1+\frac{1}{\phi\left(N_{j-1}\right)}\right) .
$$

We also write $M_{j}=N_{j}-N_{j-1}, \psi_{j}=\psi\left(N_{j}\right), D_{j}=D_{M_{j}}(n \alpha)$. Suppose $N_{k} \geq N>N_{k-1}$ and redefine $N_{k}$ if necessary to be $N$. Then the number of solutions to (10) is at least the number of solutions to

$$
\|\alpha n+\beta\|<\psi_{j}, \quad N_{j-1}<n \leq N_{j}, \quad 1 \leq j \leq k .
$$


Let $S_{j}$ be the number of solutions to (12) for one $j$. Then

$$
S_{j}=2 \psi_{j} M_{j}+O\left(D_{j}\right) .
$$

Hence, a lower bound for the number of solutions, for almost all $\alpha$, is

$$
2 \sum_{j=1}^{k} \psi_{j} M_{j}+O\left(\sum_{j=1}^{k}\left(\log M_{j}\right)^{1+\varepsilon}\right) .
$$

Now

$$
\begin{aligned}
\sum_{j=1}^{k} \psi_{j} M_{j} & \geq \sum_{n=1}^{N} \psi(n)-\sum_{j=1}^{k} M_{j}\left(\psi_{j-1}-\psi_{j}\right) \\
& =\sum_{n=1}^{N} \psi(n)+O\left(\sum_{j=1}^{k} \frac{M_{j} N_{j-1}^{-\theta}}{\phi\left(N_{j-1}\right)}\right),
\end{aligned}
$$

and

$$
\sum_{j=1}^{k} \frac{M_{j} N_{j-1}^{-\theta}}{\phi\left(N_{j-1}\right)} \ll \sum_{j=1}^{k} \frac{N_{j-1}^{1-\theta}}{\phi^{2}\left(N_{j-1}\right)} .
$$

To balance the errors in (13) and (14) we must choose

$$
\phi^{2}(n)=\frac{n^{1-\theta}}{1+(\log n)^{1+\varepsilon}},
$$

which leads to an error term of size (for $N \geq 2$ )

$$
\sum_{n=1}^{N}(\log n)^{1 / 2+\varepsilon} n^{-(1+\theta) / 2} \ll N^{(1-\theta) / 2}(\log N)^{1 / 2+\varepsilon}
$$

as required to complete the proof.

3. Proof of Theorem 2. We will give a complete proof of the case

$$
\psi(n)>\frac{\log ^{k+1+\varepsilon} n}{n^{k}}, \quad \psi(n)-\psi(m) \ll \frac{m-n}{n} \psi(n) .
$$

We will mention the modifications for the other cases later. We begin by splitting the range $1 \leq n \leq N$ into subranges as in the previous section. Put $N_{1}=1$, let $\phi_{j}=\phi\left(N_{j-1}\right)$, and write, for $j \geq 2, N_{j-1} \leq N$,

$$
N_{j}=\left[N_{j-1}\left(1+\frac{1}{\phi_{j}}\right)\right]+1
$$

If $N_{t}>N$ then put $N_{t}=N+1$. Let $\psi_{j}=\psi\left(N_{j}\right), L_{j}=\psi_{j}^{-1} \phi_{j}, M_{j}=$ $N_{j}-N_{j-1}$. Put

$$
g(L)=L^{1 / k}(\log L)^{(1+\varepsilon) / k} .
$$


Also, if $y_{j}>0$ for $j=1, \ldots, k$, let $N(L, \mathbf{x}, \mathbf{y})$ denote the number of solutions to

$$
\left\|l x_{h}\right\|<y_{h}, \quad h=1, \ldots, k, l \leq L .
$$

Lemma 1. For almost all $\mathbf{x} \in \mathfrak{M}$ we have

$$
N(L, \mathbf{x}, \mathbf{y}) \ll \prod_{h=1}^{k} \max \left(1, g(L) y_{h}\right) .
$$

Proof. Since $\mathfrak{M}$ is a Khinchin-type manifold for convergence, we have

$$
\min _{1 \leq l \leq L} \max \left(\left\|l x_{1}\right\|, \ldots,\left\|l x_{k}\right\|\right)>g(L)^{-1}
$$

for almost all $\mathbf{x} \in \mathfrak{M}$ for all sufficiently large $L$. If (15) is violated, say

$$
N(L, \mathbf{x}, \mathbf{y}) \geq T^{k} \prod_{h=1}^{k} \max \left(1, g(L) y_{h}\right),
$$

then, by the pigeon-hole principle, we can find an $l \leq L$ with, for $1 \leq h \leq k$,

$$
\left\|l x_{h}\right\| \leq \frac{c(k) y_{h}}{T \max \left(1, g(L) y_{h}\right)}<g(L)^{-1}
$$

for sufficiently large $T$. This contradicts (16) and so establishes the lemma.

Proof of Theorem 2. We shall only establish a lower bound of the form (6); the corresponding upper bound follows similarly. We have

$$
S(N, \mathbf{x}, \beta) \geq \sum_{j=1}^{t} S_{j}
$$

where $S_{j}$ counts the number of solutions to

$$
\|\mathbf{x} . \mathbf{n}+\beta\|<\psi_{j}, \quad N_{j-1} \leq \mathbf{n}^{*}<N_{j} .
$$

By a familiar argument from Fourier analysis (see [1, Chapter 2]) we have

$$
S_{j} \geq D_{j}+E_{j}
$$

where

$$
\begin{aligned}
D_{j} & =\left(2 \psi_{j}-\frac{1}{L_{j}}\right) \sum_{N_{j-1} \leq \mathbf{n}^{*}<N_{j}} 1 \\
& =\left(2 \psi_{j}-\frac{1}{L_{j}}\right) \sum_{N_{j-1} \leq n<N_{j}} k n^{k-1}\left(1+O\left(n^{-1}\right)\right),
\end{aligned}
$$

and

$$
E_{j} \ll \psi_{j} \sum_{l=1}^{L_{j}}\left|\sum_{N_{j-1} \leq \mathbf{n}^{*}<N_{j}} e(l \mathbf{n} \cdot \mathbf{x})\right| .
$$


Here $e(\alpha)=\exp (2 \pi i \alpha)$. Now, for $N_{j-1} \leq n<N_{j}$,

$$
\psi_{j}=\psi(n)-O\left(\frac{\psi(n)\left(N_{j}-n\right)}{n}\right) .
$$

Since, for $N_{j-1} \leq n<N_{j}$,

$$
\frac{N_{j}-n}{n} \ll \frac{1}{\phi_{j}} \quad \text { and } \quad \frac{1}{L_{j}} \ll \frac{\psi(n)}{\phi(n)}
$$

this gives

$$
D_{j}=2 k \sum_{N_{j-1} \leq n<N_{j}} n^{k-1} \psi(n)+O\left(\sum_{N_{j-1} \leq n<N_{j}} \frac{n^{k-1} \psi(n)}{\phi(n)}\right) .
$$

Also,

$$
E_{j} \ll \psi_{j} \sum_{l=1}^{L_{j}} \prod_{r=1}^{k}\left(\min \left(N_{j}, \frac{1}{\left\|x_{r} l\right\|}\right)\right) .
$$

We divide up the values taken by $\left\|x_{r} l\right\|$ so that either $\left\|x_{r} l\right\|<g\left(L_{j}\right)^{-1}$, or

$$
2^{s_{r}-1} \leq g\left(L_{j}\right)\left\|x_{r} l\right\|<2^{s_{r}}, \quad s_{r}=1,2, \ldots
$$

Put

$$
G_{r}= \begin{cases}N_{j} & \text { if } s_{r}=0, \\ g\left(L_{j}\right) 2^{-s_{r}} & \text { if } s_{r}>0,\end{cases}
$$

and let $\mathbf{y}=\mathbf{y}\left(s_{1}, \ldots, s_{k}\right)=g(L)\left(2^{-s_{1}}, \ldots, 2^{-s_{k}}\right)$. It follows that

$$
\begin{aligned}
E_{j} & \ll \psi_{j} \prod_{r=1}^{k}\left(\sum_{s_{r}} G_{r}\right) N(j, \mathbf{x}, \mathbf{y}) \\
& \ll \psi_{j} \sum_{r=0}^{k-1}\left(g\left(L_{j}\right) \log L_{j}\right)^{1+r} N_{j}^{k-1-r} \quad \text { by Lemma } 1 \\
& \ll \psi_{j}\left(g\left(L_{j}\right)^{k} \log ^{k} L_{j}+N_{j}^{k-1} g\left(L_{j}\right) \log L_{j}\right) \\
& \ll \frac{\psi_{j} M_{j} N_{j}^{k-1}}{\phi_{j}}\left(\frac{g\left(L_{j}\right)^{k} \phi_{j}^{2} \log ^{k} L_{j}}{N_{j}^{k}}+\frac{g\left(L_{j}\right) \phi_{j}^{2} \log L_{j}}{N_{j}}\right) .
\end{aligned}
$$

We have

$$
\frac{g\left(L_{j}\right)^{k}\left(\log L_{j}\right)^{k} \phi_{j}^{2}}{N_{j}^{k}} \ll \frac{\phi_{j}^{2}}{N_{j}^{k}} \psi_{j}^{-1} \phi_{j}\left(\log L_{j}\right)^{k+1+\varepsilon} \ll \frac{\phi_{j}^{2 k+1}\left(\log L_{j}\right)^{k+1+\varepsilon}}{N_{j}^{k} \psi_{j}}<1 .
$$

Also,

$$
\left(\frac{g\left(L_{j}\right) \log L_{j} \phi_{j}^{2}}{N_{j}}\right)^{k} \ll \frac{\phi_{j}^{2 k+1}\left(\log L_{j}\right)^{k+1+\varepsilon}}{N_{j}^{k} \psi_{j}}<1
$$


Hence

$$
E_{j} \ll \sum_{N_{j-1} \leq n<N_{j}} \frac{\psi(n) n^{k-1}}{\phi(n)} .
$$

This completes the proof on combining the ranges for $j=1, \ldots, t$.

To prove the result in the absence of condition (5) replace $\varepsilon$ by $\varepsilon / 2$ and take

$$
\phi(n)=\log \log 9 n .
$$

To complete the proof when only the condition

$$
\psi(n)>\frac{\log ^{1+\varepsilon} n}{n^{k}}
$$

is given, take $\phi(n), \varepsilon / 2$ as above, but count the solutions with weights. We note that

$$
\frac{1}{M^{2 T-1}}\left|\sum_{m=1}^{M} e(\alpha m)\right|^{2 T}=\sum_{m=-2 M T}^{2 M T} c_{m} e(\alpha m)
$$

where

$$
0 \leq c_{m} \leq C(T), \quad \sum_{m=-2 T M}^{2 T M} c_{m}=M
$$

In this section we take $T=2$ and this gives an improved estimate for $E_{j}$ in the lower bound case:

$$
\begin{aligned}
E_{j} & \ll \frac{\psi_{j}}{M_{j} N_{j}^{k-1}} \prod_{r=1}^{k}\left(\sum_{s_{r}} G_{r}^{2}\right) N(j, \mathbf{x}, \mathbf{y}) \\
& \ll \frac{\psi_{j}}{M_{j} N_{j}^{k-1}} \sum_{r=0}^{k-1}\left(g\left(L_{j}\right)\right)^{2+2 r} N_{j}^{2 k-2-2 r} \ll \psi_{j}\left(g\left(L_{j}\right)^{2 k}+N_{j}^{k-1} g\left(L_{j}\right)\right) \\
& \ll \frac{\psi_{j} M_{j} N_{j}^{k-1}}{\phi_{j}}\left(\frac{g\left(L_{j}\right)^{2 k} \phi_{j}^{3}}{N_{j}^{2 k}}+\frac{g\left(L_{j}\right)^{2} \phi_{j}^{3}}{N_{j}}\right) .
\end{aligned}
$$

This suffices to complete the proof since

$$
\frac{g\left(L_{j}\right)^{2 k} \phi_{j}^{3}}{N_{j}^{2 k}} \ll \frac{\phi_{j}^{5}\left(\log L_{j}\right)^{2+\varepsilon}}{\psi_{j}^{2} N_{j}^{2 k}} \ll 1,
$$

and similarly

$$
\frac{g\left(L_{j}\right)^{2} \phi_{j}^{3}}{N_{j}^{2}} \ll 1
$$

4. Proof of Theorem 3. The proof is, in outline, the same as the proof for Theorem 2, so we shall be very brief. We only sketch the proof of the 
case where

$$
\psi(n)^{k}>\frac{\log ^{2+\varepsilon} n}{n}, \quad \psi(n)^{k}-\psi(m)^{k} \ll \frac{m-n}{n} \psi(n)^{k} .
$$

Note that $\lambda(n)$ replaces $\phi(n)$ in our previous argument. Define $N_{j}, M_{j}, \psi_{j}$, $L_{j}$ as above, and $\lambda_{j}=\lambda\left(N_{j-1}\right)$. Lower and upper bounds are considered separately as before. For the lower bound we have

$$
T(N, \mathbf{x}, \mathbf{y}) \geq \sum_{j=1}^{t} T_{j}
$$

where $T_{j}$ counts solutions to

$$
\max _{r}\left\|n x_{r}+y_{r}\right\|<\psi_{j}, \quad N_{j-1} \leq n<N_{j} .
$$

From the method used in Section 4 of [8],

$$
T_{j} \geq 2^{k} M_{j} \psi_{j}^{k}-\frac{c(k) M_{j} \psi_{j}^{k-1}}{L_{j}}+E_{j},
$$

where

$$
E_{j} \ll \sum_{0<|\mathbf{h}|<L_{j}} \psi_{j}^{k}\left|\sum_{N_{j-1} \leq n<N_{j}} e(n \mathbf{x} . \mathbf{h})\right| .
$$

The first two terms in (17) give the main term in (8) with a suitable error. We have

$$
E_{j} \ll \sum_{0<|\mathbf{h}|<L_{j}} \psi_{j}^{k} \min \left(M_{j}, \frac{1}{\|\mathbf{x} \cdot \mathbf{h}\|}\right) \ll \psi_{j}^{k} \sum_{s \geq 0} 2^{s} N\left(s, L_{j}\right),
$$

where $N\left(s, L_{j}\right)$ counts the number of solutions to

$$
\|\mathbf{x} . \mathbf{h}\|<2^{-s}, \quad 0<|\mathbf{h}| \leq L_{j} .
$$

Since $\mathfrak{M}$ is a Groshev type manifold for convergence an application of the pigeon-hole principle (as in Lemma 1) gives

$$
N(s, L) \ll L_{j}^{k}\left(\log L_{j}\right)^{1+\varepsilon} 2^{-s} .
$$

This quickly leads to the result

$$
E_{j} \ll \lambda_{j}^{k}\left(\log N_{j}\right)^{2+\varepsilon} \ll \sum_{N_{j-1} \leq n<N_{j}} \frac{\psi(n)^{k}}{\lambda(n)}
$$

as required to complete the proof.

5. Deduction of Theorem 1. The first part of Theorem 1 follows immediately from Theorem 3 since the rational normal curve is a Groshev type manifold for convergence.

At present it is not known whether the rational normal curve is of Khinchin type for convergence (except for the case $k=2$ ). To prove the 
second part of Theorem 1 we therefore need to start with the weaker assertion (from Theorem 4) that there are only finitely many solutions to

$$
\max _{1 \leq j \leq k}\left\|\alpha^{j} n\right\|<n^{-1 / k}(\log n)^{-1-\varepsilon} \text {. }
$$

We can then modify the proof of Theorem 2, changing $g(L)$ to

$$
g(L)=L^{1 / k}(\log L)^{\varepsilon / k+1} .
$$

Following through the analysis, this changes $k+1$ to $2 k$ to complete the proof.

\section{References}

[1] R. C. Baker, Diophantine Inequalities, London Math. Soc. Monogr. (N.S.) 1, Oxford Univ. Press, 1986.

[2] V. V. Beresnevich, V. I. Bernik and M. M. Dodson, Inhomogeneous non-linear Diophantine approximation, in: Papers in honour of V. G. Sprindžuk's 60th Birthday, Inst. Math., Belarus Acad. Sci., 1997, 13-20.

[3] V. I. Bernik, Asymptotic behaviour of the number of solutions of certain systems of Diophantine inequalities, Mat. Zametki 11 (1972), 619-623 (in Russian).

[4] -, Asymptotic behaviour of the number of solutions of certain systems of inequalities in the theory of Diophantine approximations of dependent variables, Vestsi Akad. Navuk BSSR Ser. Fìz.-Mat. Navuk 1973, no. 1, 10-17 (in Russian).

[5] V. I. Bernik and M. M. Dodson, Metric Diophantine Approximation on Manifolds, Cambridge Tracts in Math. 137, Cambridge Univ. Press, 1999.

[6] J. W. S. Cassels, An Introduction to Diophantine Approximation, Cambridge Tracts in Math. and Math. Phys. 45, Cambridge Univ. Press, 1957.

[7] M. M. Dodson, B. P. Rynne and J. A. G. Vickers, Simultaneous Diophantine approximation and asymptotic formulae on manifolds, J. Number Theory 58 (1996), 298-316.

[8] G. Harman, Small fractional parts of additive forms, Philos. Trans. Roy. Soc. London Ser. A 345 (1993), 327-338.

[9] —, Metric Number Theory, London Math. Soc. Monogr. (N.S.) 18, Oxford Univ. Press, 1998.

[10] - Variants of the second Borel-Cantelli lemma and their applications in metric number theory, in: Number Theory, R. P. Bambah, V. C. Dumir and R. J. Hans-Gill (eds.), Hindustan Book Agency, 2000, 121-140.

[11] A. Khintchine [A. Khinchin], Einige Sätze über Kettenbrüche mit Anwendungen auf die Theorie der Diophantischen Approximationen, Math. Ann. 92 (1924), 115-125.

[12] W. M. Schmidt, Metrical theorems on fractional parts of sequences, Trans. Amer. Math. Soc. 110 (1964), 493-518.

Department of Mathematics

Royal Holloway, University of London

Egham, Surrey TW20 0EX, England

E-mail: G.Harman@rhul.ac.uk 